\title{
El valor institucional de la gestión pública
}

\author{
Uvalle Berrones, Ricardo* \\ * Dr. en Administración Pública (UNAM-México). Profesor a tiempo completo de la \\ Facultad de Ciencias Políticas y Sociales de la UNAM en la Licenciatura y el Postgrado en \\ Administración Pública. Investigador reconocido por el Consejo Nacional de Ciencias, \\ Tecnología y Secretaría de Educación Pública. E-mail: helmut@avantel.net
}

\section{Resumen}

El objetivo del presente artículo es analizar, categorizar y explicar a la gestión pública desde una óptica multifacética que contribuya a su revaloración institucional, para diluir la visión reduccionista que se le atribuye cuando queda confinada a las prácticas de la introspección organizativa, es decir, al mundo de las técnicas, los procedimientos y las estructuras, pero sin conectarla con el vigor de los espacios públicos de la democracia que son parte esencial de su razón de ser. Los resultados revelan que la gestión pública tiene atributos relacionados con el desempeño de los gobiernos en términos del diseño, la hechura, la implementación y la evaluación de las políticas públicas, 'por eso, al relacionar a las autoridades con los espacios privado y público, adquiere una connotación fundamental que la sitúa en el horizonte de la gobernabilidad y la estabilidad de las sociedades abiertas. Se concluye que la gestión pública es parte esencial de los procesos de gobernación, pues su aporte a la dirección implementación de las políticas es una cuestión de gran relieve que responde a los imperativos de la institucionalidad que tienen como objetivo conjugar eficacia, desempeño y calidad de vida.

Palabras clave: Gestión pública, espacio público, espacio privado, institucionalidad, gobierno.

\section{The I nstitutional Value of Public Management}

\begin{abstract}
The objective of this research is to analyze, characterize and explain public management from a multi-faceted perspective, which should contribute to institutional re-valorization, in order to dilute the reductionist vision which is assigned to it when the practice of organizational introspection is applied to the world of techniques, procedures and structures, without connecting these to the vigorous public spaces of democracy which are
\end{abstract}


an important part of their reason for their existence. The results reveal that public management has attributes related to the behavior of governments in relation to the design, creation, implementation and evaluation of public policy, and for this reason, when relating public authorities with public and private spaces, there is a fundamental connotation which is related to governability and the stability of open societies. The conclusion is that public management is an essential part of the governing process, since it offers direction in the implementation of policy and is a matter of great relevance which responds to imperatives of institutionality, the objective of which is to conjugate efficiency, fulfillment, and quality of life.

Key words: Public management, public space, private space, institutionality, government.

Recibido: 04-05-10. Aceptado: 04-11-15

\section{I ntroducción}

La importancia de la gestión pública en las sociedades democráticas y los gobiernos abiertos es incuestionable dado que es el puente que comunica y atiende la esfera privada y la esfera pública. La legitimidad de los resultados es el indicador de la calidad que tiene la propia gestión pública en los espacios sociales, políticos y económicos que son representativos de la vida productiva y amplia que se desarrolla en la esfera de lo público.

La intensidad de la participación ciudadana, el desarrollo de las economías de mercado, la comunicación entre la autoridad y los ciudadanos, las exigencias de mayor eficiencia en el desempeño institucional y el imperativo de fortalecer la gobernabilidad democrática, ubican a la gestión pública en el mundo de los procesos y los resultados que conllevan a mejorar la relación entre la sociedad y el Estado.

La relevancia de la gestión pública tiene como sustento que se relaciona con las capacidades que las instituciones gubernamentales tienen para asegurar las libertades modernas, la democracia política, la calidad de vida y el bienestar continuo. Su relevancia permite situar que sin resultados favorables de la acción de gobierno, no es posible que los ciudadanos tengan confianza en él mismo. Lo que un gobierno realiza o no, alude de modo directo a la gestión pública, ya que es el eje de las políticas públicas.

En la etapa de la crisis fiscal del Estado administrativo que deriva en el desprestigio institucional que lo aleja de la sociedad, no hay duda que la gestión pública tuvo fallas 
importantes que dan como resultado la pérdida de los consensos, la desilusión de los ciudadanos en las instituciones gubernamentales y el incumplimiento de expectativas que se aceptaron sin evaluar con realismo la capacidad instalada de las administraciones públicas.

Esta situación ocasiona una crisis de gestión debido a la operación errática de las instituciones responsables de aplicar las políticas públicas, creando de este modo, condiciones desfavorables que fermentan una crisis de confianza en los políticos y los administradores del Estado.

En este contexto, la gestión pública es revalorada de cara a las instituciones y la institucionalidad democrática para destacar que no es un referente de la instrumentalidad en sí; por contrario, es un medio que se relaciona con los valores de las instituciones y por tal motivo, su eficacia es producto no sólo de recursos técnicos y tecnológicos, sino de la orientación institucional que se imprime a los mismos en el cumplimiento de las metas públicas.

La gestión públicå1 revalorada en y por las instituciones, se transforma en un repertorio importante y complejo de operación gubernamental, dado que sus resultados tienen efecto directo en la vida social y en ese sentido, se alude a la calidad de los métodos relacionados con el cómo gobernar y cómo administrar la sociedad desde la perspectiva de los instrumentos que se aplican para desarrollar las fuerzas productivas. En este caso, los recursos y las pericias son básicos para identificar la relación directa entre la calidad de las políticas públicas y los rendimientos de las áreas económicas y sociales que configuran el modo de vida de la sociedad contemporánea.

La gestión de las políticas públicas es el epicentro de la capacidad que los gobiernos demuestran para alentar tanto el desarrollo de lo privado como de lo público. Se corrobora así, que la gestión pública tiene atributos institucionales que es indispensable considerar para que cualquier déficit de gobierno, sea contextuado en los valores de la institucionalidad, la cual permite la coexistencia de los mercados, los ciudadanos, las organizaciones y las distintas esferas gubernamentales.

Las instituciones y la institucionalidad son amplios derroteros que recorre la gestión pública para fungir como un sistema que produce y reproduce capacidades aplicadas que promueven la eficacia final de las políticas públicas. Con ello, la gestión pública debe considerarse como la gestión del gobierno, es decir, como el modo de obrar que permite articular decisiones, capacidades, recursos, estrategias, programas, operadores y acciones 
que se encaminan a la exitosa gestión de las políticas públicas.

La gestión del gobierno es un asunto de instituciones e institucionalidad, motivo por el cual, la gestión pública no debe visualizarse como una simple expresión procedimental que se encarga de la pulcra relación de los medios y los fines para responder al patrón de la racionalidad normativa que es fundamentalmente prescriptiva, es decir, autoriza, limita, incentiva y sanciona lo estipulado por los valores que postulan las normas positivas.

En otra óptica, la gestión pública entendida como gestión de gobierno, se desenvuelve en la lógica de la racionalidad heurística y en los valores de la institucionalidad democrática, que es el verdadero sentido del gobierno de lo público. El gobierno de lo público significa el gobierno por políticas y éstas se inscriben en la gestión pública porque es la responsable de ordenar, articular, elaborar, implementar y evaluar las acciones del gobierno. Por tanto, la gestión pública es el núcleo de las capacidades de gobierno y éstas son objeto de creación, es decir, son producto de las habilidades, el conocimiento y las pericias para conseguir los resultados favorables que demanda la sapiencia técnica y tecnológica para ser aplicada en el universo de las instituciones. Así, la orientación de la gestión pública es un asunto de valores negociados y de recursos utilizados para dar curso efectivo a las políticas públicas como expresión institucional de los gobiernos y las administraciones públicas.

El objetivo del presente artículo es analizar, categorizar y explicar a la gestión pública desde una óptica multifacética que contribuya a su revaloración institucional, para diluir la visión reduccionista que se le atribuye cuando queda confinada a las prácticas de la introspección organizativa, es decir, al mundo de las técnicas, los procedimientos y las estructuras, pero sin conectarla con el vigor de los espacios públicos de la democracia que son parte esencial de su razón de ser. La gestión pública tiene atributos relacionados con el desempeño de los gobiernos en términos del diseño, la hechura, la implementación y la evaluación de las políticas públicas. Por eso, al relacionar a las autoridades con los espacios privado y público, adquiere una connotación fundamental que la sitúa en el horizonte de la gobernabilidad y la estabilidad de las sociedades abiertas.

\section{Contexto}

La importancia de la gestión pública en la sociedad contemporánea refleja una de las tendencias que indican cómo las organizaciones complejas, como los Estados y las Administraciones Públicas responden a los imperativos de la vida política y económica, y cómo a través de sus medios de acción, se orientan a cumplir las metas que se han 
definido de manera institucional con la participación de una diversidad de actores que tienen interés en la atención y solución de los asuntos públicos. Hoy da, los asuntos públicos son el referente ineludible para destacar el modo de obrar de los gobiernos y las estrategias que las administraciones públicas utilizan para incorporar la voz y las energías de los ciudadanos para la atención organizada de los problemas de naturaleza pública.

En tiempos de apertura y globalidad crecientes, el dinamismo de los ciudadanos y las exigencias de la economía de mercado, son factores que obligan a que las capacidades de gobierno sean efectivas para articular demandas, intereses y soluciones que se enlazan con los requerimientos de la vida privada y la vida pública en cuanto sistemas que tienen capacidades y recursos que deben aprovecharse para diseñar respuestas inteligentes ante los problemas complejos que caracterizan a las sociedades contemporáneas.

La gestión pública es el punto medular para valorar las capacidades gubernamentales y administrativas, pues a través de ellas es posible la consecución de los fines públicos que dan vigencia, eficacia y legitimidad a los sistemas políticos que han institucionalizado los valores de la democracia. Un referente importante para la gestión pública es que se invoca por el mundo de los valores, es decir, por las instancias y las organizaciones que tienen interés en que el desempeño de los gobiernos no sea considerado una cuestión de parches o remiendos administrativos susceptibles de incrustar en alguna parte de su operación, sino que sea reivindicado como un asunto de importancia estratégica para el conjunto de la vida pública.

Los tiempos de la globalidad -modificación estructural del espacio y el tiempo con el uso intensivo de las tecnologías, las comunicaciones y los procesos de innovación- son un conjunto de retos para las organizaciones públicas, dado que las condiciones de vida son ahora más complejas e interrelacionadas. La apertura de las fronteras, la liberalización de las economías y la redefinición de las esferas estatal, privada y pública, conllevan a situaciones que favorecen más la coordinación de los esfuerzos colectivos, no tanto la dirección planificada de las políticas públicas y los programas administrativos.

La visión holística de las políticas públicas es contraria al espíritu de lo público y niega la posibilidad de que los ciudadanos puedan jugar un papel importante en los alcances de la acción de gobierno. La contundencia vertical de los programas administrativos distancia a los gobiernos de los ciudadanos, pues tienden a desplazar con sus movimientos hiperburocráticos, las aportaciones que provienen de la esfera de la vida social y ciudadana. 
Así, la gestión pública debe situarse en el universo de lo factual y de las transformaciones que vive la sociedad, sin que implique su divorcio de las formas que institucionalizan, legalizan y legitiman el poder. Distante de la valoración institucional, la gestión pública corre el riesgo de considerarse como un ejercicio instrumental y tecnocrático de la administración pública, cuando en realidad es parte activa que condiciona y contribuye en la obtención de los resultados finales. Lo institucional de la gestión pública es que se encuentra relacionada con los centros de la decisión pública, con el sistema de operación y con los sistemas de evaluación que permiten a los gobiernos el aprendizaje necesario para ser eficaces en la conducción de la sociedad.

Por tanto, la trascendencia de la gestión pública es indiscutible para comprender mejor los procesos y los resultados que se relacionan con la legitimidad de los resultados que es la mayor prueba de fuego que enfrentan los gobiernos, los políticos y los administradores

públicos. El talón de Aquiles de los gobiernos y las administraciones públicas cuando no generan resultados favorables son las fallas en la gestión pública y con ello fermentan que los índices de la legitimidad de los resultados ingresen a los caminos que tienen focos de alerta o bien focos rojos en materia de resultados conseguidos.

La gestión pública no es el confinamiento operativo de los Estados, sino el conjunto de capacidades que permiten traducir en situaciones factuales los propósitos que dan vida a las políticas y los programas de naturaleza gubernamental pero que tienen incidencia directa en la esfera de lo público. Por ello, es inexacto afirmar que la gestión pública es únicamente un asunto de procedimientos y técnicas en sí, porque su importancia llega a los diversos sitios de la sociedad en los cuales el público ciudadano juega un papel clave en su relación con el gobierno. El alcance de la gestión pública está dado por la relación directa entre la sociedad y el Estado, los ciudadanos y el gobierno y las demandas ciudadanas y las políticas públicas.

\section{El vigor de la sociedad activa}

A raíz de la crisis de las instituciones $\underline{2}$ estatales, gubernamentales y administrativas que corresponde al periodo de las políticas intervencionistas, los ciudadanos y las organizaciones reaccionan de modo contestatario para evitar mayores desplazamientos en la esfera privada y pública. La crisis del estatismo ocasiona la reacción de los ciudadanos ante las tendencias que apuntaban por una mayor injerencia de las agencias estatales en la vida de lo público.

En este sentido, la crisis de la administración pública burocrática toca fondo al no 
repuntar con ventajas comparativas que permitieran el reencuentro de los ciudadanos con el Estado. La sobrecarga de las demandas sociales y políticas no encuentra respuesta institucional eficaz para que sean encauzadas mediante la definición de agendas funcionales que faciliten su cumplimiento. La desproporción entre las demandas en competencia y la capacidad instalada de los Estados - la administración pública- ocasiona que las relaciones entre los ciudadanos y los gobiernos ingresen a zonas de desconfianza, inconformidad y resentimiento, dado que el desencanto por el deterioro de las condiciones de vida, afecta los ámbitos micro y macro sociales.

En consecuencia, la acción organizada -iniciativas, recursos, agrupaciones, estrategias y tiempos- de los ciudadanos es la respuesta para convertir a la sociedad contemporánea en una sociedad activa. Ésta se caracteriza porque se sacude la tutela estatal que le garantizaba la cultura del bienestar, pero al evaluar los costos desfavorables que ello implicaba, decide organizar sus propias capacidades para dar vigencia a formas de autonomía y autorrealización que contrarrestan las prácticas de que todo viene y proviene del Estado.

La sociedad activa es el perfil de condiciones de vida que se han replanteado los ciudadanos de manera significativa. Frente a la tutela de lo estatal se opta por la emancipación de los ciudadanos; frente al estatismo, se opta por las ventajas de la economía pública; frente a la administración pública que inhibe, se prefieren las ventajas de las políticas públicas; frente al intervencionismo magnificador y sin límites, se prefieren las políticas reguladoras, las cuales se rigen por los principios de accesibilidad, oportunidad e igualdad.

La sociedad activa es indicativa de cómo las capacidades individuales y colectivas se organizan para tener un papel más intenso en el desarrollo de la vida productiva. No ceder espacios ni iniciativas al poder sobredimensionado del Estado, es un objetivo que se proclama para ensanchar la vida pública, entendida como un espacio que pertenece a la sociedad activa y las fuerzas productivas que desarrolla para asegurar los elementos constitutivos de su vida.

Por consiguiente, los gobiernos y las administraciones públicas tienden a modificar su responsabilidad en la sociedad y la economía, optando por crear formas más abiertas de gestión pública que sean capaces de institucionalizar las expectativas y las necesidades de los ciudadanos con los recursos que la autoridad pública tiene a su cargo.

El perfil regulador de las instituciones públicas es el que empieza a prevalecer para dar 
mayor juego a las fuerzas productivas y a las organizaciones activas de la economía pública. Por tanto, el patrón de la gestión pública se orienta a los espacios abiertos de la sociedad para enlazarse con más efectividad con los actores sociales y económicos que tienen interés en participar de la producción de la riqueza material.

La gestión pública, en la visión de las políticas reguladoras, es la opción que se adopta para rearticular los ámbitos de lo privado y lo público. De este modo, el Estado es considerado fundamentalmente en las tareas de dirección y coordinación, no tanto de intervención abrumadora en la sociedad. En particular, son las tareas de coordinación las que se impulsan de manera intensa, dado que permiten que los agentes gubernamentales y los actores sociales puedan ingresar a etapas de mayor cooperación institucional, la cual es un imperativo para reactivar la eficacia y la productividad de la economía contemporánea.

El dinamismo de la sociedad abierta es el referente para situar la relevancia de la gestión pública entendida como un conjunto de actividades, procedimientos, operaciones y estrategias que tienen como objetivo asegurar la calidad y consistencia de las políticas, tomando en cuenta las aportaciones que el público ciudadano puede realizar en favor de la vida pública. El valor de la gestión pública se inserta en el mundo de las instituciones y de las organizaciones que son la plataforma para ordenar su efectividad, facilitar su aplicación y conseguir resultados espec íficos.

\section{Gestión de las Políticas}

Una faceta esencial de la gestión pública es que funge como el sustento de las políticas públicas y de la aplicación de los programas que dan vida a la cobertura de las mismas. Más allá de la visión introspectiva que es propia de la administración pública tradicional y los gobiernos cerrados, la gestión pública se desenvuelve en las instituciones y en la institucionalidad, por ser éstas las palancas que garantizan los rendimientos productivos de la sociedad y los sistemas económicos.

La aportación de la gestión pública a las instituciones consiste en que suministra los medios, los recursos y la capacidad instalada de los gobiernos para dar cumplimiento a las tareas de inter és común. En este caso, los movimientos que despliegan los gobiernos y las administraciones públicas son fruto de la gestión pública, porque es la encargada de ordenar, coordinar y producir los resultados que se encuentran en la agenda de las instituciones. 
No es causal que el nexo entre las políticas públicas y la gestión pública $\underline{3}$ tenga importancia de primer orden, porque el ámbito de lo público, es el contexto que orienta la gestación, la definición, la elaboración, la implementación y la evaluación de las políticas. Concurre en este aspecto, el modus operandi que está dado por la gestión pública y a través de ella, las políticas se convierten de un plan o programa de acción, en un conjunto de acciones, procesos y resultados que se explican por la complejidad de la vida institucional.

Al igual que las políticas públicas, la gestión pública sólo se entiende en la lógica de un gobierno por deliberación que es consustancial a los valores de la democracia. Esto significa que la categoría público-ciudadano, es la base para identificar los alcances institucionales de la gestión pública, porque sus tramos de operación no están desconectados de los valores e intereses que concurren en las políticas públicas. Éstas son un medio para organizar el comportamiento real de los gobiernos y permiten que los ciudadanos se incorporen de manera focalizada a la atención y solución de los problemas comunes.

Por ello, si las políticas públicas son la mejor expresión de un gobierno por deliberación4, la gestión pública constituye el factor más sobresaliente de cómo se da vida a los procesos de gobierno para dar cumplimiento a los valores y las necesidades que se gestan en la sociedad y que se proyectan a las instancias directivas de la vida pública. Así, el gobierno se refleja como una organización que es capaz de generar resultados para el público focalizado y su modo de obrar no es introspectivo, sino que responde a las exigencias de la sociedad activa.

Si las políticas son estrategias de gobierno, la gestión pública es la encargada de diseñarlas y aplicarlas para dar cumplimiento a los propósitos formalizados en las agendas institucionales. Corresponde a la gestión pública generar los recursos que se aplicarán en los procesos de las políticas y que se relacionan con el sistema de pericias, aptitudes y habilidades que han de demostrar los operadores del gobierno para dar coherencia al universo de las políticas públicas.

Los operadores de las políticas son los exponentes de la gestión pública porque se encargan de su implementación y eventual evaluación, con lo cual los gobiernos ingresan al terreno de las respuestas institucionales que se generan para la atención y solución de los problemas públicos. Así pues, corresponde a la gestión pública. desempeñar la tarea principal para que las políticas sean aplicadas con efectividad institucional y de ese modo mejorar la relación creciente y compleja entre los gobiernos y las organizaciones 
ciudadanas.

La gestión de las políticas es una de las tareas que tienen relevancia en los contextos de las sociedades abiertas que son el producto de los valores democráticos y del espíritu intenso de la vida pública. Se relacionan con ésta porque se identifican con el valor de lo público y con el auge del público ciudadano que son la base de los procesos que se orientan por la senda de la actividad pública.

La gestión de las políticas implica que el comportamiento de los gobiernos responde a condiciones objetivas de la sociedad, y no a la política del regateo que a veces se invoca para situar la importancia de las políticas públicas. La gestión de las políticas no se agota en patrones administrativos ni en lo administrativo; tampoco en la lógica de la dimensión técnica que únicamente valora las decisiones en sí, no su pertinencia y coherencia como productos institucionales que tendrán impacto positivo o negativo en la sociedad.

De este modo, las políticas son instrumentos que demandan pericia y sapiencia para su elaboración tomando en consideración la acción de las organizaciones y los líderes que se comportan en los diversos foros de la vida asociada. Son instrumentos de gobierno que se relacionan con las reglas que autorizan, restringen o prohíben el comportamiento de los actores sociales y políticos. No hay pues gestión de políticas sin considerar los patrones institucionales que alientan o frenan los resultados que se ofrecen en los sitios de la vida pública.

La trascendencia de la gestión pública no está en discusión, ni se ciñe a criterios de carácter instrumental que únicamente se ocupan de la relación óptima de los medios y fines. Por el contrario, es un sistema de operación qué es vital para valorar la capacidad instalada de los gobiernos y las administraciones públicas.

También se integra por valores y preferencias que reflejan cómo la vida pública es su referente indiscutible para determinar los alcances y la eficacia de las acciones públicas. Su importancia es correlativa al vigor de las instituciones y éstas cumplen sus tareas con el concurso directo de la gestión pública, dado que es la encargada de organizar la práctica de la administración pública tomando en cuenta las capacidades y los recursos de que dispone.

En cuanto conjunto de estrategias que se aplican para conseguir resultados de gobierno, la gestión pública se encarga de que las políticas públicas enlacen a la autoridad con los ciudadanos para dar tratamiento a los problemas que han ingresado a la agenda de las 
instituciones.

No obstante que la gestión pública destaca más por los medios que aporta al quehacer de las instituciones, no se queda en ese renglón, debido a que las capacidades y los recursos de que dispone, son fundamentales para determinar el grado de compromiso que el gobierno puede tener con los ciudadanos y las organizaciones representativas.

Por ello, la institucionalidad -legitimidad por resultados- de los gobiernos pierde eficacia cuando los instrumentos de la gestión pública no reúnen las condiciones óptimas para su utilización y aprovechamiento. La consistencia de las políticas públicas tiene su origen y contenido de la calidad de la gestión pública la cual es el verdadero efecto multiplicador para organizar las acciones del gobierno en la sociedad. Toda pretensión de reducir la gestión pública a los aspectos mecanicistas, operativos o instrumentales, carece de sustento porque en realidad se vincula con los valores, los objetivos y las metas que se definen en las instituciones responsables de conseguir y alcanzar los propósitos de interés común.

La misma gestión pública es portadora de valores -racionalidad técnica, racionalidad pública y racionalidad organizacional- que permiten a las instituciones de gobierno conseguir los resultados deseados y esperados. Las instituciones no operan en el vacío ni se desarrollan al margen de las presiones y las exigencias que son propias de las sociedades plurales y democráticas, sino en contextos que adoptan a la gestión pública como el pivote de los resultados que se obtienen tomando en cuenta los elementos determinantes del quehacer público.

La acción del gobierno es inexplicable sin los alcances de la gestión pública, debido a que el ámbito de la factual únicamente se manifiesta cuando los actores y los procesos se entreverán para que las políticas públicas se produzcan en los ambientes de la institucionalidad democrática. Lo valorativo y la operacional de la gestión pública se explican en razón de las instituciones y de las capacidades que acreditan para llevar a cabo la gobernación y la administración de la sociedad. Si la razón de las instituciones promotoras y reguladoras es la conservación y el desarrollo productivo de la sociedad y la economía de mercado, la razón central de la gestión pública es proporcionar las herramientas y capacidades que se conjugan para dar orden y sentido al comportamiento factual del gobierno de acuerdo con los propósitos de la vida en común.

\section{Calidad en el desempeño institucional}


Uno de los aspectos principales de la gestión pública es el relativo a la calidad de las instituciones que se encargan de crear y facilitar las condiciones que favorecen el mejor aprovechamiento de las fuerzas productivas. En efecto, la vida de las instituciones se refleja en los rendimientos que producen desde el momento en que asumen la responsabilidad de atemperar conflictos, atender demandas y decidir respuestas a una variedad de necesidades que se formulan con base en la pluralidad democrática.

Las instituciones públicas son las más comprometidas para conseguir que los resultados que obtenidos sean eficaces y congruentes con los propósitos que animan la cultura emprendedora de la sociedad. Desde la óptica de gobierno, la gestión pública es la responsable de formular los planes y las políticas que se relacionan con los capítulos más importantes de la vida productiva.

La fase de formulación exige pericia, criterio y capacidades para ordenar los cursos de acción que se consideran como factibles para estimular el desarrollo de las fuerzas productivas. Implica también, claridad de valores, objetivos y metas para definir los medios idóneos que han de permitir el cumplimiento de las políticas de acuerdo a las ofertas de gobierno.

Sin embargo, la formulación es la primera etapa que compromete las capacidades del gobierno, porque una segunda etapa compleja y determinante en lo que se propone es la implementación. La implementación se convierte en una cadena de actividades, operaciones y acciones que se encargan de dar cumplimento a los planes y políticas con base en el diseño original que los ha concebido. La implementación es un conjunto de rutas que indican los tiempos, las etapas y los procesos que sustentan la acción de los gobiernos, tomando en cuenta las situaciones previsibles y cambiantes. En este caso, la implementación es un proceso de incertidumbre que pone a prueba la capacidad instalada de los gobiernos para conseguir resultados específicos. Tanto la formulación como la implementación de los planes y las políticas, son factores esenciales en el desempeño institucional del gobierno.

El desempeño de los gobiernos es un asunto de vital importancia. No se limita al logro de la racionalidad formal -cumplimiento de las reglas y los procedimientos autorizados conforme a las normas positivas- sino que se ubica en el universo de las aptitudes que se desarrollan para asegurar, a pesar de condiciones adversas, el cumplimiento de las metas colectivas. El desempeño institucional es la clave para identificar la aceptación o el rechazo de los gobiernos por parte de los ciudadanos. 
Es en realidad, el factor que orienta si los gobernantes y los administradores públicos tienen la capacidad de producir los resultados que de ellos se espera. Incluso la relación positiva de los gobernantes y los gobernados se testimonia por la calidad del desempeño institucional, pues el mismo permite conocer si las ofertas de gobierno son congruentes con los resultados conseguidos. La comparación entre dichos elementos es de importancia creciente para que el público ciudadano valore desde diversos foros lo prometido y lo alcanzado.

Si gobernar implica eficacia para asegurar la dirección pública de la sociedad, uno de los puntos centrales es que el desempeño de las instituciones sea eficaz. Sin duda, la eficacia es parte crucial de la legitimidad, específicamente de la legitimidad por resultados. En consecuencia, el desempeño no es un asunto trivial o intrascendente, sino que ocupa un lugar básico en el horizonte de los gobiernos, pues de él depende el reconocimiento o el desprestigio que los mismos pueden tener en el corto, mediano o largo plazo. Los gobiernos son instituciones que se orientan a conseguir resultados eficaces. Tienen que ser aptos para abordar problemas, formular respuestas y mejorar de manera continua la calidad de vida en la sociedad. Por ello, el desempeño que acrediten es fundamental para determinar si la confianza de los ciudadanos en sus instituciones tiende a multiplicarse o bien corre el riesgo de ingresar a la ley de los rendimientos decrecientes.

La articulación entre desempeño institucional y gestión pública se inscribe en la lógica del modo de gobernar, es decir, en el modo de asegurar la dirección eficaz, la cual incluye la transformación positiva y fecunda de las condiciones de vida de las familias, los ciudadanos, las regiones y las comunidades. "Gobernar deja de ser el cumplimiento de reglas y normas para convertirse en la gestión de una acción colectiva cuyos agentes manejan márgenes inevitables de libertad y en la fijación de fines y resultados resulta tan problemática como la movilización de los recursos y la determinación de las consecuencias. El management deja de ser concebido principalmente como planificación y control para visualizarse ante todo como dirección, estratégica o política, o, mejor, como asunción de la responsabilidad por la acción colectiva" (Prats, 1995: 104). El desempeño es un valor relacionado no sólo con los propósitos de gobierno, sino con el logro de los resultados que se traducen en el aumento de la productividad y el bienestar social; el mejoramiento de la regulación' la consistencia de las instituciones, el cumplimiento de la equidad y el desarrollo más balanceado de las fuerzas productivas.

Cuando el desempeño es de calidad indiscutible significa que los planes y las políticas públicas son congruentes en cuanto a estructura, contenidos y acciones, lo cual es indicativo de que la gestión pública ha respondido con lo mejor de sus capacidades al 
logro de las metas colectivas. Cuando el desempeño es deficiente, la gestión pública se encuentra en posición crítica porque no acredita las capacidades que permiten a los gobiernos tener reconocimiento y aceptación. Por tanto, el declive de la gestión pública desacredita los objetivos del desempeño, y en esa medida los gobiernos se exponen a que la opinión pública y los grupos ciudadanos los señalen como instituciones que no tienen la capacidad para mejorar la calidad de vida.

Así, la disminución de la legitimidad tiene su origen en el desempeño irregular y deficiente de las instituciones gubernamentales y por ello, la relación de las autoridades con los ciudadanos ingresa a zona crítica que en términos de calendario electoral, puede tener la reacción del voto de castigo. La exigencia de la sociedad para que los gobiernos tengan un desempeño eficaz, es una de las constantes más importantes a considerar para que la gestión pública sea motivo de revisión, mejoramiento y calidad permanentes. La relación del voto de castigo y el voto de recompensa con los rendimientos de la gestión pública es correlativa. Esto significa que el desempeño institucional se ubica en las exigencias políticas y públicas de la sociedad, y por tal motivo tiene que valorarse en las decisiones estratégicas que los gobiernos han de adoptar.

Por otra parte, con la calidad del desempeño institucional también se identifica la relación entre los costos, los beneficios y las ventajas que tienen impacto en la vida pública. En este sentido, la gestión pública es el punto cardinal para destacar si los planes y las políticas guardan correspondencia en términos de proyecto, decisiones y acciones efectivas. Cuando los costos públicos -déficit fiscal, endeudamiento, sobrecarga de demandas- rebasan los beneficios y las ventajas que la sociedad puede recibir, es factible visualizar fallas en la operación del gobierno que tiene su origen en la gestión pública.

La tarea de gobierno no se acredita con sentido público y positivo cuando la sociedad carece de los elementos constitutivos de vida -libertades, democracia, participación, justicia y calidad del bienestar- que son importantes para determinar la calidad de las decisiones y acciones de gobierno. Ello implica una distancia considerable entre los valores y los hechos, lo cual significa que los planes y las políticas en cuanto expresión de las capacidades de gobierno. Tienen un déficit pronunciado que tiene impacto negativo en los centros productivos de la economía de mercado y en las áreas sociales de la vida pública.

El déficit de gobierno es sin duda un déficit significativo en la gestión pública porque ésta alude a los planes y las políticas que se aplican para conseguir resultados específicos. En consecuencia, la calidad del desempeño institucional se convierte en un mal público que tendrá repercusión extendida en la vida económica, política y social, teniendo como 
consecuencia que el prestigio del gobierno se afecte de manera considerable.

Los déficits en la gestión pública desfavorecen la calidad del desempeño gubernamental porque reflejan falta de pericia en los directivos y operadores; envían para la sociedad y los mercados señales erráticas que generan incertidumbre; desalientan a los ciudadanos en sus condiciones de vida y ocasionan que las instituciones públicas sean motivo de desprestigio.

Por tal motivo, el déficit de la gestión pública tiene que erradicarse para que los gobiernos sean nuevamente los ejes de la regulación, el desarrollo y la promoción eficaz de los agentes productivos de la sociedad. Convertir a la gestión pública en un pilar estratégico para asegurar el desarrollo pleno de la economía de mercado, es la mejor opción para evaluar la calidad del desempeño institucional. La evaluación del desempeño institucional es imprescindible en la visión que los gobiernos demuestran para asegurar la consistencia y la productividad de la vida pública.

De igual modo, convertir a la evaluación en uno de los aspectos puntuales del desempeño institucional, crea las ventajas para que la calidad de las instituciones sea un proceso ascendente porque tanto el aprendizaje de los errores, las omisiones, las deficiencias, los retrasos, las contingencia... así como de las restricciones formales e informales, son un caudal de información a resguardar y utilizar para reformular políticas, corregir desviaciones y aumentar de modo importante la eficacia constante de las instituciones.

\section{Gestión y arenas de poder}

El ambiente que se relaciona con la gestión pública aun siendo de carácter institucional, también da lugar a la existencia de arenas de poder que juegan un papel crucial en los procesos que la definen como un sistema de estrategias que se aplican para conseguir resultados específicos.

Las arenas de poder son parte de las relaciones factuales de decisión y operación que están presentes en la vida de las instituciones para influir en los ritmos y el cumplimiento de los objetivos propuestos, aluden a preferencias, intereses, motivaciones y recursos que es indispensable tener en cuenta para ubicar los recorridos que siguen los propósitos y las posibilidades que tienen como tarea impulsar a la gestión pública como productora de decisiones y acciones que se vierten a la vida de lo público en forma de planes y políticas que tendrán un tipo de impacto en la sociedad y la vida económica. 
Los procesos de gobierno toman en cuenta las ventajas de la gestión pública para dar sentido de concreción a las estrategias que se aplican en los distintos capítulos de lo público. De igual modo, los procesos de la administración pública son determinantes para orientar los rumbos de la gestión pública, pues en la propia administración pública se ubican las principales arenas de poder que están o no comprometidas con el quehacer institucional.

En este caso, los centros de la decisión burocrática son arenas de poder, ya que los protagonistas de las mismas tienen interés y valores que defienden cuando se trata de diseñar los cursos de acción del gobierno. Las arenas de poder son importantes en el desarrollo de la gestión pública, dado que son dispositivos que tienen presencia real en la selección de los medios que se utilizan para dar cumplimiento a los programas públicos.

Las arenas de poder son sistemas informales de coexistencia insertados en la vida formal de las instituciones, pero tiene un margen considerable de maniobra para apoyar o bloquear los procesos de la gestión pública. La composición de los cuadros burocráticos es un dato relevante para considerar tanto la hechura como la implementación de la gestión pública, debido a que el ambiente institucional que la legitima y legaliza, no está exento de conductas y arreglos que tienen su base en la valoración de los intereses y en las prácticas que se derivan de la política burocrática.

Sin duda, la política burocrática es un elemento vital para situar las ventajas y las restricciones de la gestión pública, ya que se integra por una variedad de conductas, valores, preferencias, procedimientos y estructuras que tienen impacto directo en el comportamiento de los gobiernos y las administraciones públicas.

La política burocrática $\underline{5}$ se ejerce en las arenas de poder y tiene como exponentes a cuadros directivos y operativos que tienen interés en el curso y las acciones que se consiguen con la gestión pública. La política burocrática es la plataforma para que las camarillas y los cenáculos vinculados con el modo institucional y factual del poder, encaucen sus acciones teniendo en cuenta el valor público de los planes y las políticas, así como los recursos que necesitan para su aplicación. Las camarillas y los cenáculos son en sí arenas de poder que tienen presencia e influencia en los derroteros de la gestión pública.

La política burocrática se manifiesta como uno de los comportamientos del gobierno y la administración pública. Es, ante todo, la suma de valores, intereses y acciones que 
sustentan el modus operandi que se relaciona con la gestión pública, dado que en ningún caso es un asunto exclusivo de dimensiones técnicas y decisionales. Tampoco es la lógica de los ejercicios pulcros que valoran a los datos y la modelación en cuanto medios que permiten tomar las decisiones óptimas soslayando los contextos donde hay relaciones activas de poder.

Sin desconocer la importancia técnica y decisional de la gestión pública, es impreciso que únicamente se le identifique por ese atributo, cuando en realidad su contenido, programas y estrategias se encuentran en los ambientes de la política burocrática.

Así pues, la política burocrática es el bastión de las arenas de poder, las cuales se integran por relaciones, estructuras, actores y estrategias que tienen como objetivo influir directamente en los procesos de la gestión pública para facilitar o bloquear las acciones a emprender. No hay comportamientos neutrales en la gestión pública, sino la expresión continua de los intereses que se enlazan con los planes y las políticas que son instrumentos del gobierno para coordinar, estimular, incentivar y apoyar los requerimientos que tienen la sociedad y la economía de mercado.

Una de las ventajas de la categoría arenas de poder es que permite identificar cómo "Los arreglos corporativistas para la elaboración y aplicación de las políticas públicas han cobrado mayor importancia a medida que los gobiernos se han visto más presionados para que adopten otros mecanismos de prestación de servicios. Cuando el "gobierno por sustitución" es más importante, los servicios pueden ser prestados por grupos de interés, por organizaciones no lucrativas, o por los métodos del sector privado, tales como la contratación. Los gobiernos se han mostrado muy activos en la promoción de los grupos y asociaciones entre el sector público y el sector privado que pueden prestar un servicio y a la vez dar legitimidad a las políticas" (Peters, 1999: 307).

Ni los técnicos ni los políticos relacionados con la gestión pública, carecen de interés en las ventajas que tiene como una herramienta que ordena, aprovecha y racionaliza los recursos escasos para tomar decisiones y conseguir resultados específicos en la sociedad. Técnicos y políticos tienen referentes valorativos que estimulan las conductas que llevan cabo y por ese motivo, la gestión pública no es ajena a las preferencias que se expresan en las arenas de poder.

La gestión pública se localiza en arenas de poder tanto formales como informales y en ningún momento es factible que se encuentren fuera de ellas. Formalmente se sitúa en la rama ejecutiva, en la administración pública y en el cuerpo de la burocracia 
gubernamental. Formalmente necesita de los recursos que autorizan los congresos o parlamentos para su aplicación y está regido por normas, estatutos, reglamentos y circulares que son la base de todo gobierno sustentado en leyes.

En el ámbito de lo formal radica la racionalidad ex ante de la gestión pública que es fundamental para organizar sus acciones futuras. En el ámbito de lo informal se localiza cómo las camarillas y los cenáculos se movilizan para asumir posiciones centrales en los procesos que conllevan a la aplicación de las estrategias que la gestión pública es capaz de producir.

Lo informal se manifiesta en los liderazgos, las promotorías, los patrocinios y los arreglos que son importantes para ubicar la racionalidad ex post de la gestión pública, ya que se relaciona de modo directo con la legitimidad de los resultados; lo informal no siempre es adverso a lo formal, incluso es una vía para alentar y asegurar la cooperación de los grupos directivos y operativos que tienen a su cargo los procesos de la gestión pública.

De este modo, la gestión pública tiene contextos políticos, estructuras de decisión y sobre todo arenas de poder que son decisivas para identificar el comportamiento institucional de los gobiernos. En este sentido, los procesos de reforma, el rediseño de las instituciones y la innovación de la gestión pública tienen que localizarse en las arenas de poder para construir los consensos que requieren, los apoyos que necesitan y los recursos que hacen posible organizarlos en etapas y programas viables. Inmersa en los procesos del poder, la gestión pública no es ajena a los mismos.

Un error frecuente es calificar la gestión pública como un ejercicio de racionalidad abstracta y técnica, cuando es un medio inmerso en el ejercicio y la distribución del poder sociablemente estructurado. El poder alude a grupos que triunfan o pierden con su ejercicio yen la lógica de ganadores y vencedores, la gestión pública es la herramienta que se utiliza para concretar la acción del gobierno.

Por ello, formal e informalmente, las arenas de poder son palancas relacionadas con la eficacia o no de la gestión pública, ya que los protagonistas de las mismas tienen la ventaja de incidir en la orientación y los alcances que conjugan los atributos écnicos, tecnológicos e institucionales de la gestión pública, con los requerimientos políticos que se traducen en apoyos, interlocución, coordinación y efectividad para conseguir el cumplimiento de las metas colectivas.

Más aún, los valores de la gestión pública relacionados con su razón de ser como son la 
eficacia, la efectividad, la productividad, la rentabilidad y la racionalidad imperfecta, tienen como núcleo de referencia las arenas de poder porque en ellas se encuentran los cuadros responsables de su dirección e implementación.

No hay pues, desconexión entre los valores de la gestión pública y los intereses de las arenas de poder que, son en última instancia, son las que influyen en el cumplimiento de uno y otros. No hay desconexión entre los valores de la gestión pública y los valores de las instituciones porque ambos se complementan en términos de los fines propuestos y los medios que se encargan de su cumplimiento. No hay desconexión entre los valores de la gestión pública y la política burocrática porque-ésta constituye el ambiente favorable o desfavorable de la gestión pública. No hay desconexión entre los valores de la gestión pública y las estructuras de decisión que se encargan de enlazar los planes y las políticas como herramientas puntuales para ordenar e implementar las acciones de gobierno.

En consecuencia, la gestión pública debe entenderse como un instrumento que condiciona o alienta el cumplimiento de metas y objetivos políticos, ya la vez como una herramienta que es condicionada en el cumplimiento de los fines públicos. En ambos casos juega un papel activo, y por tanto, no debe confinarse a prácticas instrumentales que están sujetas a los regateos y jaloneos que son propios de los "arreglos" de casino.

\section{El valor político de la gestión pública}

En el tiempo contemporáneo la gestión pública se identifica con los cambios institucionales y operativos de los Estados y las Administraciones Públicas. Si el Estado y la administración pública son sistema de poder que toman decisiones y movilizan recursos, necesitan de medios efectivos para concretar las responsabilidades que tienen a su cargo. No se gobierna sin medios efectivos ni con instrumentos que no respondan a la lógica del poder.

En este caso, para los Estados y las Administraciones Públicas la consistencia y la eficacia de la gestión pública son de gran trascendencia, porque con la misma tienen la posibilidad de cumplir tareas públicas, distribuir recursos y acercarse a la sociedad y las organizaciones que la integran. El gobierno de la sociedad es un asunto de destrezas y habilidades que se formalizan en la dirección de la misma y en el logro de las metas que se han definido. La administración de la sociedad implica la capacidad para alentar la vida privada y la vida pública con decisiones, bienes, servicios y acciones que estimulan las actividades productivas, sin perder de vista la importancia de la equidad y las relaciones de solidaridad que son propias de las comunidades que se han integrado con madurez civil 
y política.

Tanto la gobernación como la administración de la sociedad son tareas esenciales para que el valor de lo político y el valor de lo público sean los atributos que caracterizan a las sociedades abiertas y liberales. Es pues, el valor de lo político y el valor de lo público, donde se sitúa la importancia de la gestión pública, dado que se corrobora cómo que se identifica con los proyectos de la sociedad y el Estado.

La gestión pública no opera en el vacío, lo cual significa que se relaciona con ciudadanos, organizaciones, relaciones económicas, políticas, programas, formas de cooperación, sistema de estímulos, atención al público ciudadano, atención de problemas, diseño de soluciones, evaluación pública, calidad de vida, relaciones de equidad, asuntos de legitimidad, relaciones integubernamentales, gobiernos autónomos, administraciones públicas emprendedoras, corresponsabilidad social, aprovechamiento de recursos escasos, calidad de crecimiento y el desarrollo económico; en suma, se relaciona con las partes y esferas vitales de la sociedad y los comandos principales que permiten a los Estados funcionar como un sistema de respuestas organizadas y focalizadas.

En consecuencia, la gestión pública no produce políticas sin ciudadanos ni contribuye a la administración anónima de la sociedad. Produce políticas y favorece la administración de la sociedad tomando en cuenta los valores políticos y los valores públicos. Los valores políticos que son el referente para su responsabilidad social son las libertades ciudadanas, los ciudadanos activos, la participación organizada, la representación ampliada, la incorporación de los ciudadanos a la acción de gobierno. Los valores públicos que orientan su alcance son el interés general, los bienes públicos, los servicios públicos, el ciudadano como cliente, los derechos administrativos de los ciudadanos, el mejoramiento en la calidad de vida.

La gestión pública no es avalorativa ni antivalorativa; por el contrario, su contenido es valorativo y comprometido con los fines del Estado y los objetivos de la Administración pública, lo cual significa que su utilidad técnica y tecnológica se orienta al cumplimiento de fines políticos y públicos en cuanto fines que se han definido con el concurso de las autoridades y los ciudadanos. Un ángulo más contemporáneo del valor político de la gestión pública, son los procesos de rendición de cuentas, el ejercicio transparente de los recursos públicos y la evaluación pública.

Dichos aspectos se inscriben en la importancia que tienen para el régimen político la relación entre el ejecutivo y el legislativo. La gestión pública sometida a la rendición de 
cuentas es un mandato del poder Legislativo que el poder Ejecutivo debe cumplir porque emana del órgano que manifiesta la voluntad soberana de la sociedad; en este caso, el poder Legislativo.

En consecuencia, la gestión pública se ciñe a la institucionalidad democrática de la sociedad abierta y no tiene elementos para omitir que es objeto de la rendición de cuentas porque tiene a su cargo la responsabilidad y el aprovechamiento de los recursos públicos.

La gestión pública es generadora de los instrumentos técnicos y tecnológicos que se necesitan en la rama ejecutiva -la administración pública y la burocracia profesional- para mejorar los procesos de la conducción pública que implica desde luego a las políticas públicas ya los planes que ordenan las acciones específicas de la administración pública. La contribución de la gestión pública a la calidad del proceso de gobierno es indiscutible. Por eso, ella misma es motivo de las reglas que se orientan a la rendición de cuentas, la cual debe entenderse como una forma que los ciudadanos tienen para someter la administración del gobierno al escrutinio de lo público.

Por otra parte, la gestión pública no es ajena al reencuentro del gobierno con los ciudadanos, sobre todo después de que la crisis fiscal del Estado ocasiona una distancia negativa entre las autoridades electas, las autoridades designadas y el público ciudadano. El comportamiento del big goverment que fue propio de las sociedades industrializadas, desalienta la energía de los ciudadanos porque el crecimiento desmesurado de las estructuras administrativas y burocráticas, edifica barreras que tienen como costo importante, el consumo irracional del excedente económico que produce la sociedad con la participación de los agentes productivos.

El reencuentro del gobierno con los ciudadanos se inscribe en la recuperación de lo público y en el propósito de evitar que las instituciones encargadas de la dirección y la coordinación de la sociedad, no incurran en lo vicios de la cultura magnificadora que convierte al Estado en el centro único del quehacer social, como si en la sociedad no hubiera fuerzas, organizaciones y capacidades que también pueden aportar elementos importantes para la vida en común.

La cultura magnificadora empieza a sucumbir ante el renacimiento de la vida pública, la cual da cauce a otras formas de participación social que pueden aportar conocimiento, recursos, tecnologías e información para llevar a cabo la gestión de la vida productiva. Es aquí, cuando la gestión pública recobra su importancia como fórmula que permite la coordinación y la cooperación de los agentes económicos y sociales. 
La gestión pública tampoco es ajena a los procesos que conllevan a redefinir los límites de la sociedad y el Estado a consecuencia del agotamiento de patrón de la centralización, el agotamiento de las políticas intervencionistas y la insolvencia fiscal de los gobiernos. Al diluirse el monopolio de lo público en manos del Estado, se liberan energías civiles y ciudadanas que se encargan de la atención de los problemas públicos, y de ese modo, la gestión pública se erige en una salida funcional para construir las relaciones de la corresponsabilidad social.

El tránsito del Estado planificador e intervencionista al Estado regulador y promotor, es la pauta para que la gestión pública retorne de la sociedad las iniciativas que pueden aprovecharse desde lo público y que, sin oponerse al Estado, se sumen a los esfuerzos que tienden a fortalecer la esfera pública con aportaciones que son producto de la pluralidad democrática que les urge cuando la sociedad y los ciudadanos asumen un papel activo en relación a los asuntos que son de interés común.

En este caso, la nueva relación de la sociedad y el Estado ha implicado la redistribución del poder, y en esa línea, la gestión pública es el puente que comunica a los ciudadanos con el gobierno, procurando que las relaciones de cooperación entre ambos sea una de las fuentes más productivas para que las instituciones públicas no incurran en los procesos que deslegitiman al Estado y desprestigian a la administración pública.

No menos importante en cuanto al valor político de la gestión pública es que forma parte del ámbito institucional que relaciona a los gobernados con los gobernantes. La relación tradicional de la administración con los administrados que es propia de la visión monárquica del poder, cede ante la relación de la administración pública y el público ciudadano.

En este sentido, la autoridad y los ciudadanos tienen la oportunidad de convenir acuerdos de participación e intervención en la vida pública para aprovechar los recursos públicos y para compartir responsabilidades relacionadas con los problemas comunes. El acercamiento de los ciudadanos con la autoridad, es decir, de los gobernados con los gobernantes, involucra a la gestión pública en la búsqueda e institucionalización de formas de corresponsabilidad que pueden ser útiles para la atención de los requerimientos que tiene la economía de mercado.

Dichas formas de corresponsabilidad se reflejan ahora en las políticas de privatización, la subcontratación y la concesión de los servicios públicos que indican cómo la autoridad 
pública se encarga de regular y no de abdicar su responsabilidad en la vida pública.

Por otro lado, la existencia de organizaciones dedicadas a la atención de los desposeídos, al fomento de la cultura y la educación; a la defensa de los derechos civiles y políticos; al cuidado del medio ambiente; al combate de las relaciones de exclusión ya propiciar la vigencia de relaciones de solidaridad para evitar que la injusticia y la desigualdad sean un corrosivo que puede dañar la convivencia republicana $\underline{6}$, constituye uno de los pilares más destacados que testimonian cómo el vigor de lo público y de la sociedad activa es uno de los activos más valiosos para fortalecer las relaciones de comunidad que son indispensables para fortalecer el espíritu público, la convivencia social y el papel imprescindible del Estado en la defensa y vigencia de los derechos sociales.

En consecuencia, la gestión pública tambi én se vincula con los procesos que se orientan a la búsqueda de un nuevo patrón de la gobernabilidad, en este caso, de la gobernabilidad democrática 7 . En efecto, la reforma de las instituciones públicas, la conciencia sobre la importancia del dinero y los costos públicos, el control social $\underline{8}$ y democrático sobre la administración pública, la ciudadanización de los órganos de gobierno, la importancia de los resultados de los gobiernos asociando eficiencia con valores democráticos, la orientación de la administración pública a los ciudadanos, la innovación organizacional y funcional de los gobiernos, el control de los gobernados sobre los gobiernos y las nuevas relaciones de la sociedad con el Estado, son aspectos centrales en la configuración de la gobernabilidad democrática.

La médula de la gobernabilidad democrática son los procesos que conllevan a la construcción institucional de los arreglos que permiten la consolidación de la estabilidad política y el desarrollo constante de la sociedad para dotarla de mejores elementos de vida. La gobernabilidad democrática es un imperativo que exige eficacia institucional y, en este sentido, la gestión pública es portadora de los medios, los recursos y las capacidades que pueden y deben confluir en el impulso de los cambios que necesita la sociedad contemporánea y en el mejoramiento de los procesos que conllevan a la mejor gestión de las políticas y los planes que permiten incrementar la eficacia en los modos de gobernar. “... la gestión de las políticas entendidas como gestión pública, se caracteriza por articular preferencias, opciones, recursos e información para dar sentido y coherencia institucional a las demandas públicas y al conflicto de intereses. Así, las políticas son enlace funcional entre las necesidades privadas y los intereses públicos, situación que exige tratamiento de gobierno. Una característica de la sociedad de mercado es que su orientación democrática exige la participación y la representación de los actores sociales en la definición y el cumplimiento de las agendas públicas" (Uvalle, 2002: 317). 
De este modo, la gestión pública como universo diáfano en el valor de lo político y las políticas, ratifica su compromiso con las instituciones responsables de la gobernación de la sociedad activa. "En tal sentido, la misión del gobierno...es coordinar los esfuerzos privados y públicos con apego a las reglas del derecho para facilitar que su cumplimiento contribuya a tener confianza en el orden constituido. Cuando se gobierna se adoptan decisiones, lo cual implica tomar posición para negociar con los diferentes actores políticos. De ahí que la institucionalidad democrática sea la base para integrar agendas, establecer prioridades, asignar recursos y generar ventajas que sean compartidas de modo público (Uvalle, 2002: 321).

\section{Conclusión}

El horizonte de la gestión pública es la vida de las institucionales administrativas y gubernamentales que son el motor del Estado para organizar y estimular la vida de la sociedad. Los recursos que la gestión pública proporciona a los procesos del gobierno son de naturaleza técnica y tecnológica, los cuales son fundamentales para asegurar las ventajas de la acci ón organizada.

La gestión pública cobra importancia en la medida en que los contextos de la vida pública son más intensos, competitivos y plurales. Tanto la cultura de la improvisación como la cultura de los parches y los remiendos son infecundos para revitalizar las capacidades de gestión que permiten la elaboración e implementación de las políticas públicas.

Por eso, la gestión pública se erige en una estrategia que permite la consecución de resultados heurísticos para inscribirse en los procesos del gobierno. Si únicamente es considerada en su faceta instrumental, se desvaloriza como pieza central que apoya, ordena y favorece la aplicación de las políticas públicas.

Un atributo de la gestión pública consiste en agregar plusvalor al valor de los recursos utilizados porque se tiene que acreditar con la capacidad instalada exigida para la suma de habilidades y pericias que permiten desenvolverse en los ámbitos de la factibilidad de las políticas.

La gestión pública tiene como punto de referencia la utilidad institucional de los gobiernos y la capacidad de los operadores para desenvolverse en el mundo de las ventajas y las restricciones. Los procesos de gestión pública no se orientan a la austeridad 
de los recursos, sino a la economía y al aprovechamiento de los medios que posibilitan el funcionamiento real de las instituciones.

La gestión pública no se encuentra distante de los valores públicos y los valores institucionales, pues son su centro de orientación para enfatizar el uso de los recursos que tienen y que deben ir acompañados de amplias capacidades de operación, indispensables para asegurar el desempeño de los gobiernos.

En este sentido, el desempeño de los gobiernos depende de la calidad de la gestión pública y tanto la producción de las políticas públicas como de los bienes y servicios que se vierten a la sociedad, tienen como referente común el sistema de capacidades que se de muestra para influir en la obtención de los resultados finales.

El desempeño de los gobiernos es asunto estratégico en el desarrollo de las sociedades contemporáneas. La exigencia de la eficacia en la democracia es un reto para las organizaciones públicas, sobre todo para los gobiernos y las administraciones públicas. Los índices de legitimidad y la calidad de los acuerdos institucionales tienen como base cómo los gobiernos tienen la confianza de los ciudadanos a partir de su desempeño.

El desempeño refleja las capacidades y aptitudes que se tienen para gobernar y administrar la vida pública. Por ello, la gestión pública es, en los tiempos actuales, el renglón más importante para que los gobiernos se desenvuelvan con pericia en los retos de la pluralidad democrática. La eficacia de las instituciones se relaciona con el plusvalor valor agregado al valor existente- que se produce con una gestión pública competente y estratégica.

La eficacia trasciende la mera relación de fines y medios para situarse en la red de capacidades aplicados para conseguir el cumplimiento de los valores, los objetivos y las metas. La eficacia indica los modos y formas que permiten conseguir los resultados alentadores que la sociedad y la economía exigen. La eficacia es un valor relacionado con los beneficios y costos públicos que impactan la vida de los ciudadanos.

En este caso, la eficacia es parte de los valores institucionales de la gestión pública porque alude a capacidades que se han de demostrar y al cumplimiento de las metas para responder a los imperativos de la vida pública asociada. La eficacia, de acuerdo con el comportamiento de los gobiernos, se transforma de valor abstracto, en valor concreto y mensurable, es decir, ingresa al terreno de la factibilidad, los indicadores sociales y económicos, la cuantía de los recursos ya la valoración cualitativa de los programas 
públicos.

La eficacia es un valor relacionado no sólo con los fines y medios, sino con los proyectos que definen los cursos de acción del gobierno, con base en los arreglos que se consiguen entre los actores de la pluralidad democrática.

La eficacia es el arquetipo que toma en cuenta la gestión pública para conseguir resultados alentadores en el comportamiento de los gobiernos y las administraciones públicas. La eficacia es inexplicable sin los procesos de la gestión pública y constituye un referente obligado para explicar los fundamentos y los alcances del desempeño institucional.

De este modo, la gestión pública no aspira a la racionalidad exhaustiva, sino a la eficacia que reúne los aspectos cuantitativos y cualitativos que son el verdadero testimonio de cómo los gobiernos orientan sus planes y acciones para responder a la diversidad y complejidad de la vida pública. Las decisiones y las acciones de gobierno son un asunto que concierne a la gestión pública, ya que dan vida a las políticas públicas que permiten articular esfuerzos, definir sistemas de corresponsabilidad y producir resultados que indiquen las formas en que la acción pública es factible institucionalizarla a partir de los medios de colaboración entre las autoridades y los ciudadanos.

En este ángulo, la gestión pública es parte esencial de los procesos de gobernación, pues su aportación a la dirección implementación de las políticas es una cuestión de gran relieve que responde a los imperativos de la institucionalidad que tienen como objetivo conjugar eficacia, desempeño y calidad de vida.

\section{Notas}

1. “La gestión pública es un enfoque disciplinario surgido en el contexto estadunidense. Lo anterior no sugiere evidentemente que en otras realidades nacionales no existiesen las condiciones ni la necesidad de este tipo de abordaje para entender así los nuevos campos de acción y modalidades de funcionamiento de las dependencias gubernamentales. Sin embargo, resulta claro que en este país la comunidad de estudiosos de ese fenómeno, las redes de instituciones académicas y asociaciones, tanto de retóricos como de practicantes, son más amplias y dinámicas que en cualquier otro país del mundo" (Cabrero, 1998: 29).

2. "El término institucional ha llegado a significar muchas cosas diferentes. Las diferencias son importantes, pero la mayoría de la gente que habla sobre las instituciones o sobre el nuevo institucionalismo sólo comparte unas cuentas ideas clave. La noción principal es que la vida se organiza en conjunto de significados y prácticas compartidos que 
llegan a ser considerados como ciertos durante mucho tiempo. Las acciones intencionales y calculadoras de los individuos y las colectividades se encuentran insertas en esos significados y prácticas compartidas, que podemos llamar identidades..." (March y Olsen, 1997: 43).

3. “Por un lado, el enfoque de la política pública ha demostrado que los problemas públicos no son atacables sólo de manera técnica, ya que siempre existirán una multiplicidad de valores para definir el problema. De hecho, el analista de políticas no define el problema, sino que en sentido estricto lo crea. ..por otro lado, como segunda respuesta, diversos enfoques de la llamada gestión pública (public management) han constatado que el esquema institucional tradicional sobre el que actúa la administración pública conduce a la ineficiencia, no importando qué tan bien diseñado esté el sistema burocrático" (Arellano, 1999: 37).

4. “El diálogo y la discusión abierta son elementos constitutivos en la elaboración de las políticas. Esto es lo propio e irrenunciable de los regímenes democráticos. Aquí la elección y desarrollo de una política no resulta de un sofisticado cálculo tecnográfico o de una preferencia autocrática. Es, en cambio, producto de la discusión y persuasión recíproca de muchos actores políticos y gubernamentales, participantes en su formulación, aceptación y puesta en práctica". Aguilar (1997: 26).

5. “En la mayoría de las sociedades se ha creído tradicionalmente que la burocracia carece de ideas acerca de lo que deba hacer con esa misma maquinaria de gobernación que parece controlar: Sin embargo, las organizaciones burocráticas a menudo tienen sus propias ideas, bien desarrolladas, acerca de lo que debiera hacer el gobierno. Estas ideas no son declaraciones generales, como las que se encuentran en programa de un partido político, sino que se confinan al área estrecha de los conocimientos de la oficina. ..si se considera a los políticos como los amos de las ideas de políticas, la burocracia deberá considerarse como el amo de las rutinas y las técnicas" (Peters, 1999: 345 y 350).

6. “Un desafío es introducir el tema de la responsabilidad ciudadana sin diluir la responsabilidad estatal, en contra de lo propugnado por el pensamiento conservador y las políticas públicas que orientadas por aquél, han intentado justificar la reducción del papel del Estado en la promoción y protección de los derechos sociales, aduciendo que ellos son los causantes de la irresponsabilidad y pasividad social" (Cunill, 1999: 59).

7. "Desde el lado positivo, como se sabe, los argumentos en favor de la gobernabilidad de las democracias pueden ser fundados en la triple consideración de los factores de "legitimidad", "eficacia" y " estabilidad"... En principio, el gobierno democrático es el único capaz de generar una arraigada legitimidad social con base en el respeto a las libertades, al igualdad, la justicia y la tolerancia entre individuos y grupos". Camou (1995: 38).

8. “El control social es un instrumento fundamental para manejar la compleja relación entre los ciudadanos y sus agentes, los políticos, y los burócratas. Obviamente, el control de la burocracia realizado por los políticos es esencial en la democracia representativa, pero seguramente no es el único que garantiza la información que necesita la población para evaluar el carácter público de las acciones del Estado. Se ha mostrado que el control de la población sobre la 
burocracia - supervisión de tipo "alarma de incendio" es lo que asegura la mayor optimización de la relación entre el principal (ciudadanos) y sus agentes gubernamentales, toda vez que el costo de la información en este caso es menor que el que corresponde a la elación establecida entre políticos y burócratas y que las ganancias inmediatas para la sociedad son mayores" (CLAD, 1998:17).

\section{Referencias Bibliográficas}

1. Aguilar Villanueva F. Luis (1997), "Estudio Introductorio" en Evidencia, argumentación y persuasión en la formulación de políticas de Giandomenico Majone, México, Fondo de Cultura Económica.

2. Arellano Gault, David (1999) “De la administración pública a la nueva gestión: cinco dilemas", en Revista Conmemorativa del Colegio, México, Colegio Nacional de Ciencias Políticas y Administración Pública.

3. Cabrero Mendoza, Enrique (1998), “Estudio Introductorio" en La gestión pública su situación actual (coordinador) Barry Bozeman, México, Fondo de Cultura Económica.

4. Camou, Antonio (1995), "Gobernabilidad y democracia", Cuadernos de Divulgación de la Cultura Democrática, México, Instituto Federal Electoral, N úm. 6.

5. Centro Latinoamericano de Administración para el Desarrollo (1998), Una nueva gestión pública para América Latina. CLAD. Caracas.

6. Cunill Grau, Nuria (1999), “La reinvención de los servicios sociales en América Latina. Algunas lecciones de la experiencia" en Revista Reforma y Democracia, Centro Latinoamericano de Administración para el Desarrollo, Caracas, Venezuela, Núm. 13.

7. March G. James y Olsen, Johan P. (1997), “El ejercicio del poder desde una perspectiva institucional" en Revista de Gestión y Política Pública, México, Centro de Investigación y Docencia Económicas, vol. VI. Núm.1.

8. Prats i Catalá Joan, “Derecho y management en las administraciones públicas (Notas sobre la crisis y renovación de los respectivos paradigmas)" en Revista Reforma y Democracia, Centro Latinoamericano de Administración para el Desarrollo, Caracas, Venezuela, Núm. 3.

9. Peters B. Guy (1999), La política de la burocracia, México, Fondo de Cultura 
Económica.

10. Uvalle Berrones, Ricardo (2002), “Estado y mercado: Instituciones fundamentales para la gestión pública en una sociedad democrática" en Revista Venezolana de Gerencia, Universidad del Zulia, Maracaibo, Venezuela, A ño 7, N úm. 18. 